\title{
Chemical evolution models for spiral disks: the Milky Way, M31, and M33
}

\author{
Roberto D.D. Costa* \\ Instituto de Astronomia, Geofísica e Ciências Atmosféricas - USP \\ Rua do Matão 1226, 05508-090 São Paulo/SP, Brazil \\ E-mail: roberto@astro.iag.usp.br
}

Monica M. Marcon-Uchida

Instituto de Astronomia, Geofísica e Ciências Atmosféricas - USP

Rua do Matão 1226, 05508-090 São Paulo/SP, Brazil

E-mail: monicalastro.iag.usp.br

\section{Francesca Matteucci}

I.N.A.F. - Osservatorio Astronomico di Trieste

Via G.B. Tiepolo 11, 34131 Trieste, Italy

E-mail: matteucc@oats.inaf.it

The distribution of chemical abundances and their variation with time are important tools for understanding the chemical evolution of galaxies. In particular, the study of chemical evolution models can improve our understanding of the basic assumptions made when modelling our Galaxy and other spirals. We test a standard chemical evolution model for spiral disks in the Local Universe and study the influence of a threshold gas density and different efficiencies in the star formation rate (SFR) law on radial gradients of abundance, gas, and SFR. The model is then applied to specific galaxies. The model is scaled to the disk properties of three Local Group galaxies (the Milky Way, M31 and M33) by varying its dependence on the star formation efficiency and the timescale for the infall of gas onto the disk. The radial oxygen abundance gradients and their time evolution are studied in detail. The present day abundance gradients are more sensitive to the threshold than to other parameters, while their temporal evolutions are more dependent on the chosen SFR efficiency. In conclusion, we see that most massive disks seem to have evolved faster (i.e., with more efficient star formation) than the less massive ones, thus suggesting a downsizing in star formation for spirals. The threshold and the efficiency of star formation play a very important role in the chemical evolution of spiral disks. For instance, an efficiency varying with radius can be used to regulate the star formation. The oxygen abundance gradient can steepen or flatten in time depending on the choice of this parameter.

11th Symposium on Nuclei in the Cosmos

19-23 July 2010

Heidelberg, Germany.

\footnotetext{
* Speaker.
} 


\section{Introduction}

The study of the chemical evolution of nearby spiral galaxies is very important to improve our knowledge of the main ingredients used in chemical evolution models and testing the basic assumptions made in modeling our Galaxy. The other spiral members of the Local Group of galaxies, M31 and M33, have been the target of many observational studies to investigate the chemical and dynamical properties of these neighbouring systems.

In this work we present a one-infall chemical evolution model for the Galactic disk, as well as for the disks of M31 and M33. This model can predict the evolution of the abundances of 37 chemical elements from the light to the heavy ones. The model was developed assuming that the disk of each galaxy is formed by gas accretion and by varying the star formation efficiency as well as the gas accretion timescale. The similarities and the differences between the chemical evolution of these objects and the Milky Way are discussed to provide a basis for the understanding of the chemical evolution of disks.

The disks of M31 and M33 are similar to the Milky Way disk but some observational constraints, such as the present day gas distribution, can only be explained by assuming different star formation histories for these galaxies. In particular the star formation rate (SFR) is one of the most important parameters regulating the chemical evolution of galaxies together with the initial mass function.

The simultaneous reproduction of the abundance, star formation rate and surface gas density gradients along the Galactic disk can be achieved by assuming that "inside-out" disk formation occurs, as described by [1].

\section{The Chemical Evolution Model}

To reproduce the chemical evolution of the thin-disk, we adopted an updated one-infall version of the chemical evolution model presented by [2]. In this model, the galactic disk is divided into several concentric rings that evolve independently without exchange of matter. The disk is built up in the context of an "inside-out" scenario, which is necessary to reproduce the radial abundance gradients, as described by [2].

The ingredients used in the model are:

- Initial Mass Function (IMF) by Kroupa et al. [3]

- Stellar evolutionary models by Schaller et al. [4]

- Solar abundances by Asplund [5]

- Star formation rate based on the Schmidt [6] law

- Stellar yields from Woosley \& Weaver [7]

\section{Results and Discussion}

We adopt an one-infall chemical evolution model where the Galactic disk forms inside-out by means of infall of a pre-enriched gas (to take into account the effect of the halo-thick disk 
evolution), and test different thresholds and efficiencies in the SFR. The model is scaled to the disk properties of three Local Group galaxies (the Milky Way, M31 and M33) by varying its dependence on the star formation (SF) efficiency and the time scale for the infalling gas into the disk. This model is described in details by [8] and references therein.

Using this simple model we are able to reproduce most of the observed constraints available in the literature for the studied galaxies. The radial oxygen abundance gradients and their time evolution were studied in detail.

The evolution of the disk of M31 is well reproduced by assuming a faster evolution (faster means a more intense SFR which is due both to the higher efficiency of SF and to the shorter infall timescale) than in the disks of MW and M33, and also assuming a higher star formation threshold. Since the disk of M31 is more massive than those for the other galaxies, this implies that more massive disks should form faster. It should be noted that the threshold and the efficiency in the star formation play a very important role in the chemical evolution of spiral disks and a variable efficiency along the radius can be used to regulate the star formation. The oxygen abundance gradient can steepen or flatten in time depending on the choice of this parameter.

Concerning the SF threshold effect in the radial oxygen gradients we note that it is more visible for the Milky Way and M33 than for M31. This fact is compatible with the scenario proposed by [9] who suggest that the SF threshold can produce a truncation in the observed stellar luminosity profile of spiral discs and that low-mass galaxies should have smaller values for this radius than the massive ones.

In figure 1, we show the results for the oxygen abundance gradient for the Milky Way (left upper panel), M31 (right upper panel), and M33 (left lower panel) with a compilation of observational data and a comparative plot between the predictions for the galaxies (right lower panel). The abundance results are slightly shifted for the MW and overestimated for M33, but the predicted slopes of the gradients are in agreement with the observational data.

Figure 2 shows the evolution of the oxygen abundance gradient with time. Models are the same as those present in Figure 1. It is not easy to establish whether the radial abundance gradient tends to flatten or steepen with time: the models predict either flattening or steepening of the gradient depending on the adopted SFR along the disk. As one can see, for ?(R) all models predict a gradient that flattens with time.

\section{Conclusions}

Our results can be summarized as follows:

- Star formation threshold effect is more visible for the MW and M33. This fact is compatible with the suggestion of Pholen et al. (2004) who says that the threshold can produce a truncation in the observed stellar luminosity profile of spiral disks and that low mass galaxies should have smaller values for this radius than the massive ones.

- The present day chemical abundance gradients for the oxygen predicted by the models are in good agreement with observational data. Figure 1 shows that the slope of the gradient changes for distinct input parameters, and for distinct galactocentric distances. For the Milky 

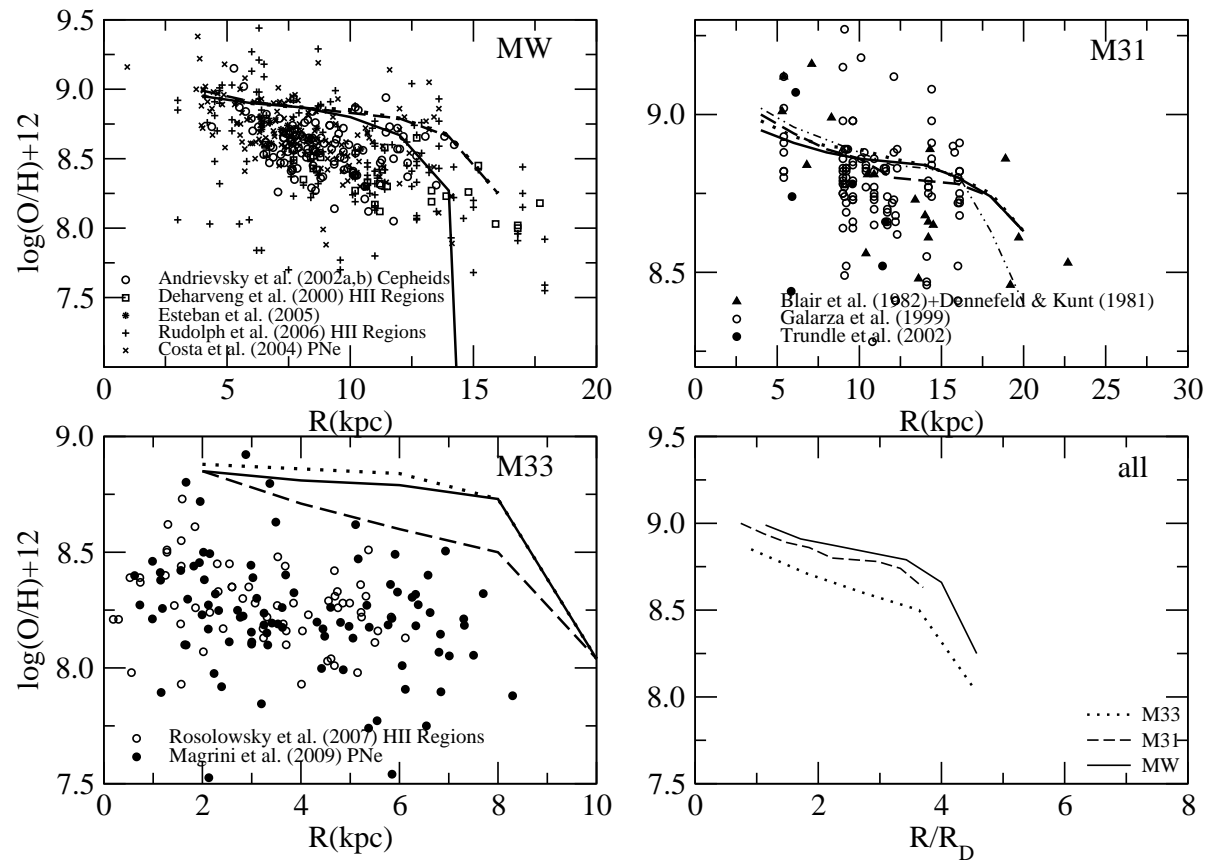

Figure 1: Radial oxygen abundance gradient for the three galaxies in the sample. In these figures, solid, dashed and dot-dashed lines represent different model outputs. See [8] for the detailed references on symbols and the origin of the observational data. The lower right panel shows the model prediction with variable efficiencies in the star formation process for all galaxies, using the normalized radius (solid line represents the MW, dashed M31, and dotted M33).

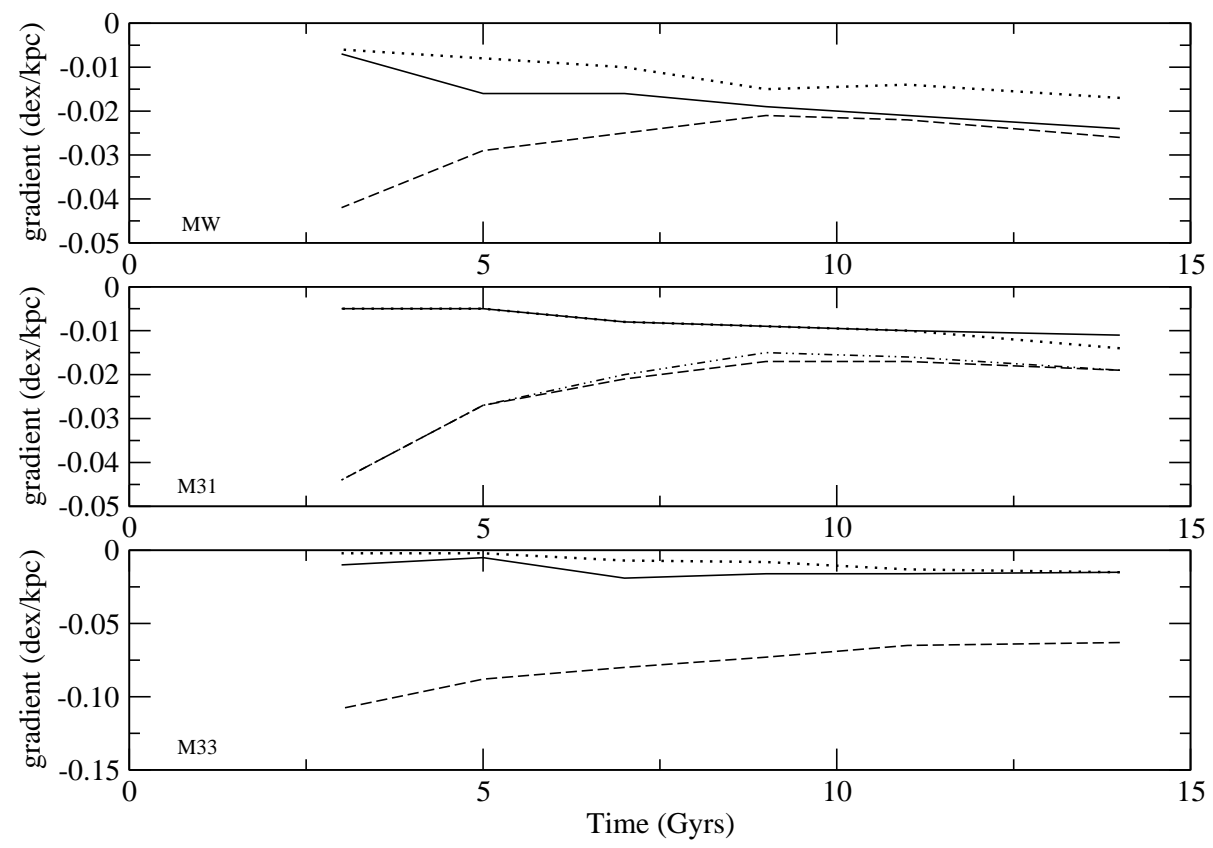

Figure 2: Time evolution of the slope of the radial abundance gradient of oxygen for all models. The first panel shows the results for the Milky Way (solid, dotted and dashed lines represent different models), while the middle panel is for M31 and last panel shows the predictions for M33, also for different model parameter sets (see [8] for details). 
Way it can be seen that models with star formation efficiency depending on the galactocentric distance $R$ present a steeper gradient, closer to the observational data.

- The time evolution of the gradients can assume different trends depending on the star formation efficiency adopted.

- For all galaxies the gas profile is better reproduced by the models with variable SF efficiency. For the MW the observational data show a peak near $5 \mathrm{kpc}$ and a decrease outwards, and the results are in good agreement with observations. For M31 the data show a strong peak at 12 $\mathrm{kpc}$ that is also better reproduced with the SF efficiency varying through the disk. The models fail to reproduce the gas density in the inner region of M33, whose gas profile displays a continuous decrease outwards. This is probably due to some bulge-disk interaction. See [8] for further details.

- Comparing the distributions of the present day stellar density in all three galaxies we note that it gets flatter from M33 to M31, indicating a possible correlation between the galaxy total mass surface density and the slope of the stellar distributions.

- The most massive disks seem to have evolved faster (i.e., with more efficient star formation) than the less massive ones, thus suggesting a downsizing in star formation for spirals.

\section{References}

[1] E. Colavitti, G. Cescutti, F. Matteucci, G. Murante, 2009, The origin of abundance gradients in the Milky Way: the predictions of different models,A\&A 496, 429.

[2] C. Chiappini, F. Matteucci, D. Romano, 2001, Abundance Gradients and the Formation of the Milky Way, ApJ 554, 1044.

[3] P. Kroupa, C.A. Tout, G. Gilmore, 1993, The distribution of low-mass stars in the Galactic disc, MNRAS 262, 545.

[4] G. Schaller, D. Schaerer, G. Meynet, A. Maeder, 1992, New grids of stellar models from 0.8 to 120 solar masses at $Z=0.020$ and $Z=0.001, A \& A S$ 96, 269 .

[5] M. Asplund, N. Grevesse, A.J. Sauval, 2005, The Solar Chemical Composition, in Cosmic Abundances as Records of Stellar Evolution and Nucleosynthesis, ASP Conf. Ser. 336, 25.

[6] M. Schmidt, 1963, The Rate of Star Formation. II. The Rate of Formation of Stars of Different Mass, ApJ 137, 758.

[7] S.E. Woosley, T.A. Weaver, 1995, The Evolution and Explosion of Massive Stars. II. Explosive Hydrodynamics and Nucleosynthesis, ApJS 101, 181.

[8] M.M. Marcon-Uchida, F. Matteucci, R.D.D. Costa, 2010, Chemical evolution models for spiral disks: the Milky Way, M31, and M33, A\&A 520, A35.

[9] M. Pohlen, J.E. Beckman, S. Hüttemeister, J.H. Knapen, P. Erwin, R.-J. Detmar, 2004, Stellar Disk Truncations: Where do we stand?, in: Penetrating bars through masks of cosmic dust : the Hubble tuning fork strikes a new note, ASSL 319, 713 